\title{
Comparação dos hábitos pessoais e conhecimento basal de promoção de saúde entre alunos do primeiro ano do Curso de Especialização de Medicina do Trabalho (Turma 2011), alunos residentes e internos do quinto ano da Faculdade de Medicina da Universidade de São Paulo
}

\author{
Comparison of personal habits and baseline knowledge of health promotion \\ among students of the Faculty of Medicine, University of São Paulo
}

\author{
Clarissa Mari de Medeiros ${ }^{1}$, Renata Bittar Brito Arantes ${ }^{2}$, \\ Eduardo Costa Sá $^{3}$, Mário Ferreira Junior ${ }^{4}$
}

\begin{abstract}
Medeiros CM, Arantes RBB, Sá EC, Ferreira Junior M. Comparação dos hábitos pessoais e conhecimento basal de promoção de saúde entre alunos do primeiro ano do Curso de Especialização de Medicina do Trabalho (Turma 2011), alunos residentes e internos do quinto ano da Faculdade de Medicina da Universidade de São Paulo/Comparison of personal habits and baseline knowledge of health promotion among students of the Faculty of Medicine, University of São Paulo. Rev Med (São Paulo). 2014 jan.-mar.;93(1):41-51.
\end{abstract}

RESUMO: Durante os anos de graduação do curso de medicina pouca ênfase é dada à importância da promoção e prevenção de saúde na vida dos médicos e pacientes. Este trabalho foi desenvolvido para avaliar o conhecimento basal dos alunos de especialização em medicina do trabalho sobre promoção de saúde e correlacioná-lo com o conhecimento basal dos alunos da Faculdade de Medicina da Universidade de São Paulo (internos do quinto ano e residentes do primeiro ano de clínica médica) além de avaliarmos os seus próprios hábitos de saúde. Para isto fizemos uma revisão bibliográfica de 1997 a 2011 sobre o tema, nas bases de dados online. Foi realizado um estudo transversal no qual foram aplicados dois questionários que já haviam sido utilizados em estudos anteriores por Ferreira Junior, 2011. Os questionários forma denominados 1) "Questionário de atitudes e Comportamento" e 2) "Questionário de avaliação de conhecimento em promoção de saúde". No questionário 1 as médias menores dos alunos da pós-graduação se apresentaram nos itens uso de cigarro e drogas, situações de estresse, comportamento sexual, qualidade do sono, vacinação e check up atuais, sendo que a expectativa para o próximo ano se manteve menor nos itens uso de drogas, estresse e prevenção de acidentes e violência. As notas maiores entre os pós-graduandos, foram relacionadas à alimentação e higiene bucal, tanto no momento atual como a expectativa para daqui um ano. As questões sobre atitude profissional, nos seus 13 itens as notas dos pós-graduandos foram maiores no item Capacitação. Nos demais itens, as notas dos alunos da graduação e da residência médica, e dos alunos da pós-graduação foram iguais. Quando comparadas as médias dos alunos da pós-graduação no chamado questionário 2, todos os itens apresentaram valores menores em relação às notas de internos e residentes. De acordo com os resultados obtidos observamos certo menosprezo com os temas relacionados a promoção da saúde. O prejuízo na qualidade do atendimento é inegável, uma vez que não há consciência por parte dos entrevistados da própria falta de conhecimento técnico atualizado com relação a promoção da saúde que contrasta com a auto-percepção de capacitação adequada para fazê-lo.

DESCRITORES: Promoção da saúde; Prevenção de doenças; Medicina preventiva; Saúde do trabalhador; Local de trabalho; Comportamentos saudáveis; Ambiente de trabalho.

ABSTRACT: During the years of the undergraduate medical course little emphasis is given to the importance of health promotion and prevention in the lives of doctors and patients. Typically, the teaching is based on a curative medicine. But, nowadays, the implementing health promotion programs are

\footnotetext{
${ }^{1}$ Preceptora do Programa de residência de Medicina do Trabalho e Medicina Legal da FMUSP. E-mail: clarissamari@hotmail.com.

2. Médica do trabalho formada pela residência de Medicina do Trabalho da FMUSP.

3. Médico do SSO-HCFMUSP, Professor do curso de Especialização em Medicina do Trabalho do Departamento de Medicina Legal, Ética Médica, Medicina Social e do Trabalho da Faculdade de Medicina da Universidade de São Paulo. E-mail: eduardocs6@gmail. com.

4. Médico do AGD-HCFMUSP, Professor Dr. em Patologia do Trabalho pela USP.

Endereço para correspondência: Clarissa Mari de Medeiros. Instituto Oscar Freire - FMUSP. Rua Teodoro Sampaio, 115 - São Paulo, SP. E-mail: clarissamari@hotmail.com.
} 
important to generate significant positive results for society and especially for businesses, employees and the financial market. This study aims to increase the sample size of a monograph made in 2011 and evaluate health promotion knowledge of first year's students of specialization in Occupational Medicine, correlating it with the knowledge of the second year's students of specialization in Occupational Medicine of Medicine College of São Paulo University, after Health Promotion module, and evaluate their own health habits. We'll do a literature review from 1997 to 2014 on the topic in the online databases. A cross-sectional study will be done

\section{INTRODUÇÃO}

$\mathbf{T}$ Trabalhar com saúde, e não com doença ou sequelas é possível e deve ser feito sempre. A prevenção e promoção de saúde além de gerar melhor qualidade de vida aos assistidos diminuem expressivamente a incidência de patologias e mortes ${ }^{1}$.

Pensar em prevenção e promoção de saúde é essencial, principalmente dentro do ambiente de trabalho, pois a saúde é determinada pelas condições de vida e trabalho dos seres humanos e só pode ser pensada em um cenário em que haja um dimensionamento social. Já que a dinâmica da produção, das condições do labor e do modo de vida é a principal fonte para a compreensão do processo de saúde/ doença e morte dos trabalhadores ${ }^{1}$.

Nas últimas décadas, observamos que há uma ênfase progressiva do ensino de promoção de saúde, assim como de prevenção de doenças, no currículo dos cursos de especialização de medicina do trabalho, devido principalmente à sua relevância na diminuição da incidência de acidentes, doenças do trabalho, absenteísmo, presenteísmo* assim como uma melhora da qualidade de vida, longevidade, produtividade, autonomia e independência.

A compreensão pelo médico do trabalho da forma de organização do trabalho associada com as condições de vida do trabalhador é necessária para que haja uma reformulação da proposta de gestão em saúde do trabalhador nas empresas ${ }^{1}$.

Este entendimento não deve partir apenas do médico do trabalho, mas de todos os profissionais que lidam diretamente com o trabalhador, incluindo o empregador. Há uma necessidade de integralização de setores como saúde, trabalho e emprego que consigam contemplar ações de prevenção, promoção, vigilância, assistência e reabilitação que garantam ao funcionário um atendimento humano e integralizado ${ }^{1}$.

Para que isto ocorra, as ações precisam da participação das empresas no estímulo à implementação de pro- with two questionnaires that had been used in previous studies by Ferreira Junior 2011. Questionnaires are called 1) "Survey of attitudes and behavior" and 2) "Questionnaire of knowledge will be held in promoting health.” Then, statistical analysis will be made with data obtained by questionnaires and through the evaluation of results we'll conclude the study.

KEYWORDS: Health promotion; Disease prevention; Preventive medicine; Occupational health; Workplace; Health behavior; Working environment. gramas de promoção de saúde que também visem garantir condições salubres de trabalho e que ajudem na eliminação dos riscos à saúde do trabalhador ${ }^{1}$.

Um estudo realizado por Serxner et al. ${ }^{2}$ demonstrou que a associação entre os programas de promoção de saúde e os programas de redução de risco gera maiores benefícios à população de trabalhadores do que quando realizados individualmente ${ }^{2}$.

Estas são propostas que redefinem o papel das empresas na participação na política pública de saúde e de sua responsabilidade na promoção de saúde ${ }^{1}$.

\section{Correlação do conhecimento dos médicos sobre promoção de saúde com os seus próprios hábitos pessoais de saúde}

Um fato que precisa ser ressaltado diz respeito às poucas publicações sobre a correlação de hábitos saudáveis dos médicos com seu interesse de fazer orientações de promoção de saúde aos seus pacientes. O pressuposto é que os profissionais mais saudáveis podem cuidar melhor da saúde de seus pacientes. Além disso, os médicos que têm hábitos saudáveis pessoais são mais propensos a incentivar os pacientes a adotar hábitos semelhantes ${ }^{3}$.

\section{Avaliação da importância do ensino de Prevenção e Promoção de Saúde}

Devido à importância dada a implementação dos Programas de Promoção de Saúde nos dias atuais gerando resultados positivos importantes para a sociedade e principalmente para as empresas, os funcionários e o mercado financeiro este trabalho foi desenvolvido para avaliar o conhecimento basal dos alunos de especialização em medicina do trabalho sobre promoção de saúde e correlacioná-lo com o conhecimento basal dos alunos da FMUSP (internos do quinto ano e residentes do primeiro ano de clínica médica)

\footnotetext{
* Presenteísmo: Presença física e ausência mental. Refere-se a estar presente fisicamente no trabalho, mas sem ação e comprometimento)
} 
além de avaliarmos os seus próprios hábitos de saúde para que possamos verificar se há a necessidade de estimularmos ainda mais o ensino da disciplina de Promoção de Saúde assim como a de prevenção de doenças no currículo do Curso de Medicina e da Especialização de Medicina do Trabalho.

\section{OBJETIVO}

Realizar um estudo populacional dos alunos pósgraduandos lato sensu do primeiro ano do curso de Medicina do Trabalho avaliando as variáveis: gênero, idade, estado civil e tipo de escolaridade.

Avaliar os hábitos de vida e os conhecimentos médicos de promoção e prevenção de saúde destes alunos (do primeiro ano de Medicina do Trabalho da Universidade de São Paulo - Turma 2011) e comparar os mesmos com resultados de estudo anterior realizado com alunos da graduação e do primeiro ano da Residência de Clínica Médica.

\section{MÉTODOS}

\section{Revisão bibliográfica}

A revisão bibliográfica sobre o tema consistiu na consulta de artigos disponíveis nas bases de dados online (Pubmed, SciELO, Lilacs) com os descritores em português e inglês: Promoção de saúde no local de trabalho (worksite health promotion), Prevenção de doenças (disease prevention), Medicina preventiva (preventive medicine), Proteção da saúde no local de trabalho (workplace health protection), Segurança e saúde ocupacional (occupational safety and health), Produtividade (productivity), Comportamento saudável (healthy behavior), bem como livros e revistas especializadas.

Para inclusão neste trabalho foram considerados os artigos relacionados ao tema publicados de 1997 a 2011, inicialmente através da leitura dos resumos e posteriormente a leitura na íntegra dos trabalhos que tinham relação com o tema e com os objetivos propostos neste estudo.

\section{Desenho do estudo}

Realizamos um estudo transversal com pós-graduandos ingressos no primeiro ano do Curso de Especialização em Medicina do Trabalho da Faculdade de Medicina da Universidade de São Paulo, em 2011.

\section{Questionários}

\section{Aplicação dos Questionários}

Utilizamos para a pesquisa dois questionários elaborados para aplicação em estudos anteriores ${ }^{3,4}$, devidamente aprovados pela Comissão de Ética para Análise de Projetos de Pesquisa (CAPPesq) da Faculdade de Medicina da Universidade de São Paulo.

No início do curso, as pesquisadoras fizeram uma explanação breve aos Pós-graduandos de Medicina do Trabalho sobre o objetivo da pesquisa em questão, convidandoos a participar. Os que concordaram assinaram o Termo de Consentimento Livre e Esclarecido (TCLE).

O primeiro questionário aplicado foi denominado pelos autores de: "Questionário de atitudes e Comportamento" e o segundo "Questionário de avaliação de conhecimento em promoção de saúde".

Precisamos ressaltar que os questionários foram aplicados antes da realização do Módulo de Promoção de Saúde do Curso de Pós-graduação lato sensu de Medicina do Trabalho (que é aplicado apenas no segundo ano do curso) para que pudéssemos avaliar as atitudes, comportamento pessoal e o conhecimento básico dos alunos com relação à promoção de saúde e prevenção de doenças.

\section{Descrição dos questionários}

\section{Questionário de atitudes e Comportamento}

No questionário de atitudes e comportamento os alunos deveriam responder a perguntas de identificação como nome, gênero, idade, estado civil e tipo de escola que estudaram durante o ensino médio (pública ou privada).

Posteriormente, foram solicitados a responder perguntas quanto aos hábitos de vida como consumo de álcool, tabaco e drogas, realização de atividade física, hábitos alimentares, enfrentamento do estresse, comportamento sexual, higiene oral, sono, exposição à radiação ultra violeta (UV), prevenção de lesões, imunização e realização rotineira de exames de check up.

As perguntas foram objetivas e avaliaram a percepção do aluno quanto à adoção pessoal de hábitos saudáveis assim como a importância que os mesmos dão ao estímulo dos bons hábitos aos seus pacientes. Para respondê-las os candidatos precisavam respeitar a graduação das 11 opções dadas que variavam de zero (muito ruim) a dez (muito bom).

As perguntas avaliavam quatro aspectos: 1) Os hábitos de saúde atuais; 2) Expectativa para os mesmos hábitos de saúde após um ano da aplicação do questionário; 3) Suas habilidades atuais no fornecimento de orientação para os seus pacientes; 4) Seu interesse de fazer orientações de promoção de saúde aos seus pacientes.

\section{Questionário de avaliação de conhecimento em promoção de saúde}

O questionário de avaliação de conhecimento em promoção de saúde era composto por quarenta questões sobre temas variados de promoção de saúde e prevenção de doenças.

As perguntas foram divididas de acordo com a im- 
portância de cada objeto da educação médica assim como da prática clínica. Elas foram determinadas e subdivididas pela equipe multidisciplinar do Centro de Promoção de Saúde do Hospital das Clínicas da Faculdade de Medicina da Universidade de São Paulo (HC-FMUSP).

As questões foram divididas em três categorias conforme ilustrado na Tabela 1: 1) Assunto da pergunta: Questões que envolviam conceitos em promoção de saúde e prevenção de doenças, rastreamento, aconselhamento e quimioprofilaxia; 2) Tipo de conhecimento exigido na per- gunta: Questões de natureza teórica e prática; 3) Nível de dificuldade da pergunta: Questões com nível de dificuldade fácil, média e difícil.

As duas primeiras categorias foram assim definidas pelo autor do questionário ${ }^{4}$. A terceira categoria foi definida pelo desempenho dos alunos do quinto ano da Faculdade de Medicina da USP, sendo as fáceis as 10 perguntas com maior nível percentual de acerto, as difíceis as 10 perguntas com maior nível percentual de erro e as outras foram classificadas como intermediárias ${ }^{4}$.

Tabela 1 - Composição do questionário de avaliação de conhecimento em promoção de saúde

\begin{tabular}{lll}
\hline Assunto da pergunta & Número das perguntas & Quantidade (\% do total) \\
\hline Conceitos de Promoção de Saúde & $21,24,26,27,28$ & $5(12,5 \%)$ \\
\hline Rastreamento & $2,3,9,10,12,23,25,29,32,33,39$ & $11(27,5 \%)$ \\
\hline Aconselhamento & $4,5,6,8,11,13,15,16,17,18,19,20,22,30,31$, & $19(47,5 \%)$ \\
\hline Quimioprofilaxia & $34,36,37,38$ & $5(12,5 \%)$ \\
\hline Tipo de conhecimento exigido na pergunta & Número das perguntas & Quantidade (\% do total) \\
\hline Conteúdo Teórico & $1,2,3,4,5,17,20,21,22,24,26,27,28,37$ & $14(35 \%)$ \\
\hline \multirow{2}{*}{ Conteúdo Prático } & $6,7,8,9,10,11,12,13,14,15,16,18,19,23$, & $26(65 \%)$ \\
\hline Nível de dificuldade da pergunta & $25,29,30,31,32,33,34,35,36,38,39,40$ & \\
\hline Fáceis & Número das perguntas & \multirow{2}{*}{ Quantidade (\% do total) } \\
\hline Intermediárias & $13,14,15,16,18,26,27,28,29,34$ & $10 \%)$ \\
\hline Difíceis & $1,6,7,8,9,11,12,19,20,21,22,23,30,31,32$, & $20(50 \%)$ \\
\hline
\end{tabular}

\section{Estatística descritiva}

Com relação aos dados epidemiológicos, estes foram submetidos à análise estatística descritiva com avaliação de média, mediana e desvio padrão da idade e porcentagem simples de gênero, estado civil e tipo de ensino médio.

Para o questionário de atitudes e comportamento, utilizamos o questionário feito por Ferreira Junior et al.,4 que utilizou o critério alfa de Crombach para dar confiabilidade e consistência interna ao mesmo. Este índice é muitas vezes recomendado para avaliar instrumentos de pesquisa psicométricas. Um coeficiente de confiabilidade maior ou igual a 0,70 é considerado aceitável em pesquisa científicas. Para este questionário os cálculos do alfa de Crombach foram próximos de 1.0, indicando, portanto, que o questionário reflete bem as atitudes e o comportamento dos alunos. Comparando as médias obtidas neste estudo, com as notas apresentadas na aplicação do questionário em alunos do quinto ano da graduação e residentes de clínica médica do primeiro ano, observamos, de maneira geral, diferenças importantes que permitem aventar hipóteses interessantes sobre o comportamento do médico no decorrer de sua formação profissional.

A comparação entre os resultados do nosso questionário de atitudes e comportamento e o questionário do estudo prévio de Ferreira Junior et al. ${ }^{3}$ foi feito a partir das médias das notas obtidas em ambos os estudos.

Para o questionário de avaliação de conhecimento em promoção de saúde utilizamos os filtros da tabela excel para definirmos por categorias de questões descritas a média das médias de acertos e o desvio padrão das respostas certas.

A comparação entre os resultados do nosso questionário de conhecimento em promoção de saúde e o questionário do estudo prévio de Ferreira Junior et al. ${ }^{4}$, foi feito a partir das médias das notas obtidas em ambos os estudos. 


\section{RESULTADOS}

Dos 100 artigos analisados, depois de aplicados os critérios de inclusão considerando apenas os artigos relacionados ao tema publicados de 1997 a 2011, a partir da leitura de seus resumos e posteriormente da íntegra daqueles que tinham relação com o tema e com os objetivos propostos neste estudo, e excluídos artigos mais antigos e em línguas não dominadas pelos autores deste trabalho, restaram apenas 39 .

Com relação aos questionários, dos 60 alunos matriculados no $32^{\circ}$ curso de pós graduação de Medicina do Trabalho, no ano de 2011, apenas 29 (48\%) concordaram em participar da pesquisa, respondendo às perguntas de ambos os questionários.

Tabela 3 apresenta a percepção do grupo de estudo a respeito de seus próprios hábitos de vida e saúde, no momento atual e uma estimativa para um ano após esta data, além de uma avaliação subjetiva do seu nível de interesse e capacitação para orientar pacientes sobre assuntos de promoção da saúde e prevenção de doenças. A Tabela 4 apresenta a percepção do grupo-referência utilizada para fins comparativos, neste estudo. Mostra os valores médios, e respectivos desvios-padrões no grupo de internos e residentes, submetidos ao mesmo questionário, em estudo anterior. A Tabela 5 compara os dados obtidos neste estudo com os dados apresentados por Ferreira et al. ${ }^{3}$.

Tabela 2 - Características demográficas do grupo de estudo $(\mathrm{N}=29)$

\begin{tabular}{lcc}
\hline Idade & Média (Desvio Padrão) & $\mathbf{3 5 , 0}(\mathbf{8 , 8})$ \\
\hline Gediana & $\mathbf{3 1 , 0}$ \\
\hline Masculino & $\mathbf{N}$ & $\mathbf{\%}$ \\
\hline Feminino & 15 & 51,7 \\
\hline Total & 14 & 48,3 \\
\hline Estado Civil & $\mathbf{2 9}$ & $\mathbf{1 0 0}$ \\
\hline Solteiro (a) & $\mathbf{N}$ & $\mathbf{\%}$ \\
\hline Casado (a) & 18 & 62,1 \\
\hline Divorciado (a) & 6 & 20,7 \\
\hline Viúvo (a) & 4 & 13,8 \\
\hline Sem Resposta & 0 & 0,0 \\
\hline Total & 1 & 3,4 \\
\hline Tipo de Ensino & $\mathbf{2 9}$ & $\mathbf{1 0 0 , 0}$ \\
Escolaridade & $\mathbf{N}$ & $\mathbf{9}$ \\
\hline Público & 6 & 20,7 \\
\hline Privado & 19 & 65,5 \\
\hline Público/Privado & 1 & 3,4 \\
\hline Sem Resposta & 3 & 10,3 \\
\hline Total & $\mathbf{2 9}$ & $\mathbf{1 0 0 , 0}$ \\
\hline
\end{tabular}

Tabela 3 - Auto percepção dos alunos do $32^{\circ}$ ano do curso de especialização em Medicina do Trabalho quanto a seus hábitos pessoais e seu comportamento profissional em relação a assuntos de promoção a saúde e prevenção de doenças. Média (desvio padrão) em uma escala de 0 (péssimo ou muito baixo) a 10 (ótimo ou muito alto)

\begin{tabular}{lllll}
\hline & \multicolumn{1}{c}{ Comportamento Pessoal } & \multicolumn{2}{c}{ Atitude Profissional } \\
\hline Bebida alcoólica & & $\begin{array}{c}\text { Expectativa para } \\
\text { daqui a 1 ano }\end{array}$ & Capacitação & Interesse \\
\hline Cigarro/tabaco & $8,1(2,1)$ & $8,6(1,7)$ & $7,8(1,4)$ & $7,0(2,3)$ \\
\hline Drogas & $9,2(2,1)$ & $9,8(0,5)$ & $8,5(1,3)$ & $7,9(2,6)$ \\
\hline Atividade física & $9,2(2,2)$ & $9,6(1,3)$ & $7,6(1,7)$ & $7,1(3,3)$ \\
\hline Alimentação & $5,3(3,4)$ & $8,7(1,5)$ & $8,5(1,1)$ & $8,7(1,5)$ \\
\hline Situações de stress & $6,9(1,8)$ & $8,7(1,2)$ & $8,4(1,3)$ & $8,6(1,8)$ \\
\hline Sexo & $6,7(1,8)$ & $8,4(1,5)$ & $7,3(1,3)$ & $7,0(2,3)$ \\
\hline Higiene bucal & $8,0(2,2)$ & $9,4(1,0)$ & $7,3(1,7)$ & $6,3(2,6)$ \\
\hline Sono (qualidade e horas) & $8,2(1,8)$ & $9,5(0,8)$ & $7,2(2,2)$ & $6,1(3,1)$ \\
\hline Exposição ao sol (e outras fontes UV) & $7,5(2,0)$ & $8,7(1,7)$ & $7,1(2,0)$ & $6,6(2,7)$ \\
\hline Violência/acidentes & $7,7(1,9)$ & $8,9(1,4)$ & $6,1(1,7)$ & $7,2(2,8)$ \\
\hline Check-up & $5,1(3,3)$ & $8,6(2,0)$ & $7,4(1,5)$ & $7,5(2,3)$ \\
\hline Vacina & $7,4(3,3)$ & $8,7(2,6)$ & $7,2(1,9)$ & $7,2(2,3)$ \\
\hline
\end{tabular}


Medeiros CM et al. Comparação dos hábitos pessoais e conhecimento basal de promoção de saúde entre alunos.

Tabela 4 - Referência: Auto percepção dos alunos da graduação e alunos da residência médica - primeiro ano no "Questionário de atitudes e Comportamento". Média (desvio padrão) em uma escala de 0 (péssimo ou muito baixo) a 10 (ótimo ou muito alto)

\begin{tabular}{|c|c|c|c|c|c|c|c|c|}
\hline \multirow[t]{3}{*}{ REFERÊNCIA } & \multicolumn{2}{|c|}{$\begin{array}{l}\text { Comportamento } \\
\text { Pessoal }\end{array}$} & \multicolumn{2}{|c|}{ Atitude Profissional } & \multicolumn{2}{|c|}{$\begin{array}{l}\text { Comportamento } \\
\text { Pessoal }\end{array}$} & \multicolumn{2}{|c|}{ Atitude Profissional } \\
\hline & \multicolumn{4}{|c|}{ Residentes } & \multicolumn{4}{|c|}{ Internos } \\
\hline & Atual & $\begin{array}{l}\text { Próximo } \\
\text { ano }\end{array}$ & Capacitação & Interesse & Atual & $\begin{array}{l}\text { Próximo } \\
\text { ano }\end{array}$ & Capacitação & Interesse \\
\hline Bebida alcoólica & $8,3(1,6)$ & $8,7(1,5)$ & $6,9(1,3)$ & $8,2(1,5)$ & $7,9(1,9)$ & $8,4(1,9)$ & $7,1(1,4)$ & $8,6(1,7)$ \\
\hline Cigarro/tabaco & $9,3(2,1)$ & $9,7(1,3)$ & $7,5(1,4)$ & $9,2(1,1)$ & $9,7(1,1)$ & $9,9(0,3)$ & $7,7(1,6)$ & $9,0(1,5)$ \\
\hline Drogas & $9,8(1,3)$ & $9,8(1,3)$ & $6,5(2,1)$ & $8,7(1,2)$ & $9,6(1,2)$ & $9,8(0,7)$ & $6,5(2,2)$ & $8,8(1,7)$ \\
\hline Atividade física & $4,6(2,8)$ & $8,1(2,4)$ & $7,5(1,5)$ & $9,2(1,0)$ & $6,0(3,0)$ & $8,1(2,0)$ & $7,8(1,7)$ & $9,0(1,2)$ \\
\hline Alimentação & $6,0(1,9)$ & $8,3(1,4)$ & $7,3(1,6)$ & $9,0(1,2)$ & $6,7(1,8)$ & $8,3(1,1)$ & $7,1(1,8)$ & $8,8(1,6)$ \\
\hline $\begin{array}{l}\text { Situações de } \\
\text { estresse }\end{array}$ & $6,9(1,3)$ & $8,4(1,5)$ & $6,0(2,0)$ & $7,3(1,9)$ & $6,8(2,0)$ & $8,6(1,5)$ & $5,3(2,5)$ & $7,7(2,5)$ \\
\hline $\begin{array}{l}\text { Comportamento } \\
\text { sexual }\end{array}$ & $8,4(1,9)$ & $9,2(1,0)$ & $7,6(1,7)$ & $7,9(2,1)$ & $8,7(1,7)$ & $9,6(0,7)$ & $6,7(2,1)$ & $8,1(2,1)$ \\
\hline Higiene bucal & $7,7(1,4)$ & $9,0(1,4)$ & $6,5(2,5)$ & $6,5(2,7)$ & $8,5(1,3)$ & $9,2(1,1)$ & $6,7(2,4)$ & $7,2(2,7)$ \\
\hline Sono & $8,4(1,9)$ & $6,8(2,2)$ & $7,2(1,7)$ & $8,0(2,1)$ & $6,7(1,7)$ & $7,5(2,0)$ & $6,7(2,1)$ & $7,7(2,3)$ \\
\hline Exposição ao sol & $7,7(1,4)$ & $8,3(1,7)$ & $7,8(1,6)$ & $7,8(1,9)$ & $7,4(1,9)$ & $8,5(1,3)$ & $7,9(1,8)$ & $8,3(2,1)$ \\
\hline $\begin{array}{l}\text { Violência /Aci- } \\
\text { dentes }\end{array}$ & $5,7(2,0)$ & $9,0(1,3)$ & $6,1(1,8)$ & $7,3(2,4)$ & $8,7(1,3)$ & $9,2(1,1)$ & $6,3(2,6)$ & $8,0(2,3)$ \\
\hline Check up & $5,7(3,4)$ & $7,6(2,8)$ & $7,7(1,4)$ & $8,9(1,4)$ & $5,6(3,3)$ & $7,6(3,0)$ & $6,3(2,3)$ & $8,6(1,7)$ \\
\hline Vacina & $8,8(2,1)$ & $9,3(1,8)$ & $7,4(1,5)$ & $8,8(1,4)$ & $9,0(1,5)$ & $9,7(0,6)$ & $6,6(2,1)$ & $8,8(1,8)$ \\
\hline
\end{tabular}

Tabela 5 - Média das notas dos alunos da graduação e da residência médica, comparadas às médias obtidas com alunos da pós-graduação - primeiro ano, no "Questionário de atitudes e Comportamento" Média (desvio padrão) em uma escala de 0 (péssimo ou muito baixo) a 10 (ótimo ou muito alto)

\begin{tabular}{|c|c|c|c|c|c|c|c|c|}
\hline & \multicolumn{4}{|c|}{ Comportamento Pessoal } & \multicolumn{4}{|c|}{ Atitude Profissional } \\
\hline & \multicolumn{2}{|c|}{ Atual } & \multicolumn{2}{|c|}{$\begin{array}{l}\text { Expectativa para daqui } \\
\text { a } 1 \text { ano }\end{array}$} & \multicolumn{2}{|c|}{ Capacitação } & \multicolumn{2}{|c|}{ Interesse } \\
\hline & $\begin{array}{l}\text { Pós gra- } \\
\text { duandos }\end{array}$ & $\begin{array}{l}\text { Média } \\
\text { internos e } \\
\text { residentes }\end{array}$ & $\begin{array}{l}\text { Pós gra- } \\
\text { duandos }\end{array}$ & $\begin{array}{l}\text { Média } \\
\text { internos e } \\
\text { residentes }\end{array}$ & $\begin{array}{l}\text { Pós gra- } \\
\text { duandos }\end{array}$ & $\begin{array}{l}\text { Média } \\
\text { internos e } \\
\text { residentes }\end{array}$ & $\begin{array}{l}\text { Pós gra- } \\
\text { duandos }\end{array}$ & $\begin{array}{l}\text { Média } \\
\text { internos e } \\
\text { residentes }\end{array}$ \\
\hline Bebida alcoólica & $8,1(2,1)$ & 8,1 & $8,6(1,7)$ & 8,5 & $7,8(1,4)$ & 7,0 & $7,0(2,3)$ & 8,4 \\
\hline Cigarro/tabaco & $9,2(2,1)$ & 9,5 & $9,8(0,5)$ & 9,8 & $8,5(1,3)$ & 7,6 & $7,9(2,6)$ & 9,1 \\
\hline Drogas & $9,2(2,2)$ & 9,7 & $9,6(1,3)$ & 9,8 & $7,6(1,7)$ & 6,5 & $7,1(3,3)$ & 8,7 \\
\hline Atividade física & $5,3(3,4)$ & 5,3 & $8,7(1,5)$ & 8,1 & $8,5(1,1)$ & 7,6 & $8,7(1,5)$ & 9,1 \\
\hline Alimentação & $6,9(1,8)$ & 6,3 & $8,7(1,2)$ & 8,3 & $8,4(1,3)$ & 7,2 & $8,6(1,8)$ & 8,9 \\
\hline Situações de stress & $6,7(1,8)$ & 6,8 & $8,4(1,5)$ & 8,5 & $7,3(1,3)$ & 5,6 & $7,0(2,3)$ & 7,5 \\
\hline Sexo & $8,0(2,2)$ & 8,5 & $9,4(1,0)$ & 9,4 & $7,3(1,7)$ & 7,1 & $6,3(2,6)$ & 8,0 \\
\hline Higiene bucal & $8,2(1,8)$ & 8,1 & $9,5(0,8)$ & 9,1 & $7,2(2,2)$ & 6,6 & $6,1(3,1)$ & 6,8 \\
\hline $\begin{array}{l}\text { Sono (qualidade e } \\
\text { horas) }\end{array}$ & $5,5(2,6)$ & 7,5 & $8,4(1,6)$ & 7,1 & $7,1(2,0)$ & 6,9 & $6,6(2,7)$ & 7,8 \\
\hline $\begin{array}{l}\text { Exposição ao sol (e } \\
\text { outras fontes UV) }\end{array}$ & $7,5(2,0)$ & 7,5 & $8,7(1,7)$ & 8,4 & $8,1(1,7)$ & 7,8 & $7,2(2,8)$ & 8,0 \\
\hline Violência/acidentes & $7,7(1,9)$ & 7,2 & $8,9(1,4)$ & 9,1 & $6,7(1,6)$ & 6,2 & $6,5(2,8)$ & 7,6 \\
\hline Check-up & $5,1(3,3)$ & 5,6 & $8,6(2,0)$ & 7,6 & $7,4(1,5)$ & 7,0 & $7,5(2,3)$ & 8,2 \\
\hline Vacina & $7,4(3,3)$ & 8,9 & $8,7(2,6)$ & 9,5 & $7,2(1,9)$ & 7,0 & $7,2(2,3)$ & 8,8 \\
\hline
\end{tabular}


Quanto ao questionário da avaliação do conhecimento em promoção de saúde dos alunos da pós-graduação, após utilizarmos os filtros da tabela Excel, encontramos os seguintes valores descritos na Tabela 6. A Tabela 7 é uma referência retirada de estudo semelhante com alunos residentes e internos do HCFMUSP ${ }^{4}$. A Tabela 8 compara os resultados entre o nosso estudo e o estudo realizado em 2011 por Ferreira Junior et al. ${ }^{4}$.

Tabela 6 - Desempenho dos alunos no questionário de avaliação de conhecimento em promoção de saúde (N=29)

\begin{tabular}{lcc}
\hline Categorias das Questões & Número de Questões & Média (Desvio padrão) de respostas corretas \\
\hline Avaliação Completa & 40 questões & $47,8(6,6)$ \\
Conceitos & 5 questões & $49,6(7,0)$ \\
Rastreamento & 11 questões & $31,0(5,8)$ \\
Aconselhamento & 19 questões & $53,9(6,4)$ \\
Quimioprofilaxia & 5 questões & $59,3(3,4)$ \\
Conteúdo Teórico & 14 questões & $41,4(6,8)$ \\
Conteúdo Prático & 26 questões & $51,2(6,4)$ \\
Fáceis & 10 questões & $73,1(3,4)$ \\
Intermediárias & 20 questões & $48,4(4,0)$ \\
Difíceis & 10 questões & $21,0(3,5)$ \\
\hline
\end{tabular}

Tabela 7 - Referência: Desempenho dos alunos da graduação (primeiro e quinto ano) e residentes (primeiro ano) da FMUSP em 2011 no questionário de avaliação de conhecimento em promoção de saúde ${ }^{4}$

\begin{tabular}{lcccc}
\hline Categorias das Questões & Número de Questões & $\begin{array}{c}\text { Y1 } \\
(\mathbf{N}=\mathbf{1 7 2})\end{array}$ & $\begin{array}{c}\text { Y5 } \\
(\mathbf{N}=\mathbf{7 6})\end{array}$ & $\begin{array}{c}\text { R1 } \\
(\mathbf{N}=\mathbf{5 6})\end{array}$ \\
\hline Avaliação Completa & 40 questões & $45,0(8,6)$ & $64,9(8,6)$ & $72,9(10,2)$ \\
Conceitos & 5 questões & $55,9(12,4)$ & $74,2(18,1)$ & $76,0(15,9)$ \\
Rastreamento & 11 questões & $21,3(9,3)$ & $51,7(13,9)$ & $64,4(16,5)$ \\
Aconselhamento & 19 questões & $53,2(11,8)$ & $67,4(9,59)$ & $75,5(10,4)$ \\
Quimioprofilaxia & 5 questões & $55,3(23,0)$ & $75,0(17,9)$ & $78,5(15,6)$ \\
Conteúdo Teórico & 14 questões & $44,4(11,5)$ & $56,4(11,8)$ & $60,8(12,9)$ \\
Conteúdo Prático & 26 questões & $45,3(10,7)$ & $71,8(10,4)$ & $79,4(10,6)$ \\
Fáceis & 10 questões & $76,6(15,6)$ & $93,4(9,0)$ & $94,8(8,5)$ \\
Intermediárias & 20 questões & $42,0(11,5)$ & $69,6(13,1)$ & $78,6(12,8)$ \\
Difíceis & 10 questões & $19,4(11,7)$ & $12,9(13,8)$ & $39,6(18,2)$ \\
\hline
\end{tabular}

Tabela 8 - Média das notas dos alunos da graduação (Y) e da residência médica (R), comparadas às médias obtidas com alunos da pós-graduação (P) no "Questionário de avaliação de conhecimento em promoção de saúde" Média (desvio padrão) em uma escala de 0 (péssimo ou muito baixo) a 100 (ótimo ou muito alto)

\begin{tabular}{lccccc}
\hline & $\begin{array}{c}\text { Internos } \\
(\mathbf{Y})\end{array}$ & $\begin{array}{c}\text { Residentes } \\
(\mathbf{R})\end{array}$ & $\begin{array}{c}\text { Média entre } \\
\text { Y/R }\end{array}$ & $\begin{array}{c}\text { Pós Graduandos } \\
(\mathbf{P})\end{array}$ & $\begin{array}{c}\text { Diferença entre as } \\
\text { médias P e Y/R }\end{array}$ \\
\hline Avaliação completa & $64,9(8,6)$ & $72,9(10,2)$ & 68,9 & 47,8 & 21,1 \\
Conceitos & $74,2(18,1)$ & $76,0(15,9)$ & 75,1 & 49,6 & 25,5 \\
Rastreamento & $51,7(13,9)$ & $64,4(16,5)$ & 58,0 & 31,0 & 27,0 \\
Aconselhamento & $67,4(9,5)$ & $75,5(10,4)$ & 71,4 & 53,9 & 17,5 \\
Quimioprofilaxia & $75,0(17,9)$ & $78,5(15,6)$ & 76,7 & 59,3 & 17,4 \\
Conteúdo teórico & $56,4(11,8)$ & $60,8(12,9)$ & 58,6 & 41,4 & 17,2 \\
Conteúdo prático & $71,8(10,4)$ & $79,4(10,6)$ & 75,6 & 51,2 & 24,4 \\
Fáceis & $93,4(9,0)$ & $94,8(8,5)$ & 94,1 & 73,1 & 21 \\
Intermediárias & $69,6(13,1)$ & $78,6(12,8)$ & 74,1 & 48,4 & 25,7 \\
Difíceis & $26,9(13,8)$ & $39,6(18,2)$ & 33,2 & 21,0 & 14,2 \\
\hline
\end{tabular}




\section{DISCUSSÃO}

Comparando as médias obtidas neste estudo, com as notas apresentadas na aplicação do questionário em alunos do quinto ano da graduação e residentes de clínica médica do primeiro ano, observamos, de maneira geral, diferenças importantes que permitem aventar hipóteses relevantes sobre o comportamento do médico no decorrer de sua formação profissional.

Fogaça et al. ${ }^{5}$, discorre sobre a qualidade de vida de médicos em formação, residentes e profissionais da área em diversas especialidades. É possível concluir que médicos (...) apresentam altos esforços, demandas psicológicas, físicas e insegurança no trabalho que repercutem na qualidade de vida). Sabe-se que diversos itens são pertinentes ao conceito de qualidade de vida - alimentação adequada, qualidade do sono e horas de sono, prática de atividade física regular, abolir/evitar consumo excessivo de álcool bem como hábitos de consumo de drogas e cigarro. Bons hábitos, como praticar atividade física, ter tempo para lazer, e não fumar estão associados à melhor avaliação da saúde em geral e devem ser incentivados ${ }^{6}$. A satisfação profissional também teve um peso importante no bem-estar emocional relatado pelos participantes do estudo de Torres et al. ${ }^{6}$, no qual foi proposto autoavaliação sobre qualidade de vida e saúde física e mental por egressos na Faculdade de Medicina da UNESP.

Neste trabalho foram pesquisadas questões relacionadas ao consumo de bebida alcoólica, alimentação, atividade física, uso de drogas e cigarro, situações de estresse, comportamento sexual, higiene bucal, qualidade do sono, exposição à luz solar, prevenção de acidentes e violência, vacinação e os chamados atendimentos e exames de check up.

O consumo de bebida alcoólica, avaliado neste trabalho, além de revelar diferenças inerentes à personalidade de cada aluno, apresentou variações expressivas no intervalo numérico referente à nota atribuída ao próprio consumo. A nota eleita mostra o que o aluno acredita ser adequado (ou não) ao seu próprio consumo do álcool. Assim sendo, a nota 10 não foi atribuída, necessariamente, ao consumo zero, o que poderia ser considerado ideal num estudo de entrevista de médicos, e sim o grau de satisfação pessoal com o próprio consumo da bebida alcoólica. Os alunos da pós-graduação apresentam grau de satisfação pessoal com valor médio de 8,1 . Os transtornos relacionados ao consumo de álcool são (...) alarmantes e responsáveis por mais de $10 \%$ de todos os problemas de saúde no Brasil ${ }^{17}$. Educação sobre os efeitos do álcool, aconselhamento básico sobre como ficar abstinente ou diminuir o consumo, monitoramento das complicações médicas relacionadas e acompanhamento ambulatorial feito pelo clínico são eficazes na redução da morbidade e da mortalidade relacionadas ao álcool. O presente estudo mostrou que a auto avaliação quanto a capacidade em orientar sobre o consumo de ál- cool apresentou valor médio de 7,8 e a nota do interesse no assunto, com média de 7,03.

O consumo próprio atual de cigarro e drogas foi avaliado de maneira similar, com nota média de 9.27 para ambos. O assunto mostra a relevância da saúde de quem cuida da saúde, uma vez que diversos transtornos clínicos e da saúde mental estão diretamente relacionados ao uso e abuso de tabaco e drogas. Para Fidalgo e Silveira ${ }^{7}$, em 2008 , o questionamento sobre o assunto para a classe médica resultou nos seguintes dados: 60,2\% acreditam que os médicos são mais suscetíveis ao uso abusivo de psicotrópicos quando comparados à população geral. No entanto, $88,0 \%$ do total consideram difícil a procura por ajuda especializada. Estudos anteriores mostraram em amostra de 11.909 médicos, $759(6,4 \%)$ são fumantes regulares e $11.150(93,6 \%)$ são não fumantes; 4.085 médicos $(34,3 \%)$ são ex-fumantes ${ }^{8}$.

E em relação à atividade física, a nota obtida foi uma das menores da avaliação, com média de 5,37, com aumento expressivo na expectativa para o próximo ano, valor médio de 8,74 . No estudo realizado por Macedo et al. ${ }^{9} \mathrm{em}$ 2009 , apenas $32 \%$ dos residentes entrevistados realizavam alguma atividade física regular. Silva et al. ${ }^{10}$, afirma em seu trabalho que se acredita na atividade física como uma forma de restaurar a saúde dos efeitos nocivos que a rotina estressante do trabalho/estudo traz (...). A conclusão de seu estudo revela que dentre as diferenças na qualidade de vida das pessoas que praticam atividades físicas comparadas com as que não praticam, não estão apenas os aspectos de saúde física, mas também aspectos psicológicos e cognitivos. Atividade física é tema frequente de recomendação médica, e os alunos da pós-graduação avaliaram o seu nível de capacitação e de interesse em fazê-lo, respectivamente, pelas médias 8,51 e 8,79.

A questão que leva em consideração a alimentação, também revelou médias baixas - atualmente 6,98 com estimativa de 8,75 para o próximo ano. Não apenas a questão nutricional e sua consequência direta na saúde física do indivíduo são contemporizadas na questão: sabe-se que transtornos endócrinos, cardiovasculares e psiquiátricos podem ocorrer pela alimentação inadequada. A obesidade é um dos grandes fatores responsáveis pela diminuição da capacidade cardiorrespiratória e, principalmente, musculoesquelética dos acometidos, aumentando a incidência de doenças crônicas ${ }^{11}$. Indivíduos com transtornos alimentares apresentam maiores prejuízos na maioria dos escores de qualidade de vida, especialmente no que diz respeito ao aspecto mental ou domínios que o compõem ${ }^{12}$. A capacitação técnica avaliada pelos alunos da pós-graduação revela média de 8,48 . O interesse nessa orientação mostrou valor médio de 8,68.

Segundo Firth-Cozens ${ }^{18}$, citado por Torres et al. ${ }^{6}$, a prevalência de médicos que apresentam estresse acima da média situa-se em torno de $28 \%$, tanto em estudos longitudinais quanto transversais. (...) apesar das inúmeras 
gratificações (...), a profissão médica costuma ser altamente estressante e ansiogênica. Distúrbios depressivos também não são infrequentes devido ao estresse, o que pode desencadear, além de prejuízos de âmbito pessoal, efeitos no ambiente de trabalho. De acordo com Williams et al. ${ }^{13}$, há uma perda de mais de 5 horas de produtividade, por semana, dos indivíduos portadores de depressão. A capacidade de enfrentar situações com estresse avaliada neste estudo apresentou média atual de 6,77 e aumento para 8,48, como expectativa para o próximo ano. Orientar o enfrentamento de situações de estresse foi também avaliado pelos alunos da pós-graduação, mostrando média de 7,37 na capacitação técnica em aconselhar maneiras de atenuar estresse média de 7,06 no interesse dessa abordagem.

Bianco et al. ${ }^{14}$, em seu estudo sobre impacto das condições bucais na qualidade de vida, traz conceitos em relação a diversos itens ou domínios associados a melhora ou piora nas atividades cotidianas. Questionamentos em relação à dor, limitação funcional, desconforto psicológico, incapacidade física, social e psicológica relacionadas às condições bucais devem ser realizados para que a completa contemporização da qualidade de vida do paciente seja realizada. A higiene bucal apresentou média de 7,27 na capacitação por parte dos alunos da pós-graduação e valor médio de 6,10 no interesse da orientação de preservação dentária, escovação, uso de próteses, e seus cuidados, entre outros assuntos referentes ao tema. $\mathrm{Na}$ auto avaliação deste item relacionado à higiene bucal, os alunos da pós-graduação apresentaram média de 8,2 no momento da entrevista e 9,55 na expectativa para próximo ano.

Os distúrbios do sono provocam consequências adversas na vida das pessoas por diminuir seu desempenho diário, aumentar a propensão a distúrbios psiquiátricos, déficits cognitivos (...), e por comprometer a qualidade de vida $^{15}$. Em estudo realizado em 2006, citado por Lourenção et al. ${ }^{16}$, em sua revisão bibliográfica, $38 \%$ dos residentes entrevistados se consideravam excessivamente sonolentos e $7 \%$, muito sonolentos. A qualidade do sono e as horas diárias de sono obtidas pelos pós-graduandos mostraram média de 5,59. No entanto, os pós-graduandos mostraramse otimistas com a melhora do sono, mostrando média estimada para o próximo ano de 8,41. A nota relativa a essa capacitação, nos pós-graduandos entrevistados, mostrou média de 7,17 e interesse de 6,65 em média.

$\mathrm{O}$ item relacionado ao comportamento sexual se refere a cuidados em relação à prevenção de doenças sexualmente transmissíveis e gravidez indesejada. De acordo com Kurakawa et al. ${ }^{19}$, em meio à diversidade dos desafios trazidos pela epidemia do HIV/AIDS (do inglês, Human immunodeficiency virus infection / Acquired immunodeficiency syndrome), a assistência à saúde dos indivíduos acometidos constitui uma de suas facetas mais problemáticas. A complexidade e a variedade dos problemas suscitados pela AIDS exigem respostas por parte dos serviços de saúde, que considerem não somente os aspectos clínicos, mas também os impactos sociais, psicológicos e econômicos associados aos estigmas e preconceitos que ainda a permeiam. A nota obtida à capacitação na orientação de comportamento sexual foi, em média, de 7,31 e interesse de 6,31 pelos alunos da pós-graduação. $\mathrm{O}$ valor médio absoluto relacionado ao comportamento sexual dos próprios médicos, ou seja, a nota atribuída aos hábitos dos profissionais da saúde em relação ao uso correto de contraceptivos e preservativos mostrou valores menores quando comparados ao estudo anterior, realizados em residentes e alunos da graduação média atual de 8,03.

O papel da vacina, inquestionável em diversas doenças infecciosas presentes no calendário vacinal, mantém sua importância durante a vida adulta, devendo ser questão sempre abordada uma vez que se trata de ação coletiva e individual de saúde. Os profissionais da saúde têm risco aumentado para doenças imunopreveníveis, com possibilidade de transmissão intra-hospitalar, e risco significativo de contraí-las e/ou transmiti-las, conforme sua susceptibilidade $^{20}$, devendo, desta forma, apresentar calendário vacinal atualizado sempre, para a sua segurança e a de seus pacientes. Estudo realizado em hospital universitário da cidade de São José do Rio Preto em 2006 revela que dos 375 alunos (59,8\%) entrevistados, apenas 69 alunos (11,0\%) apresentaram espontaneamente a carteira vacinal e nenhuma das carteiras apresentou-se em dia com as vacinas de rotina do adulto e dos profissionais de saúde ${ }^{20}$. $\mathrm{O}$ estudo concluiu que são imperativos planejamento e ação em saúde regular, normatizada na instituição estudada e demais escolas de ensino superior, para proteção da população suscetível. No presente estudo, o auto cuidado no setor da saúde obteve nota de 7,48 dentre os entrevistados. A conclusão do estudo de Rio Preto pode ser reproduzida nesse item.

Outro item relacionado à prevenção de doenças e promoção da saúde é a exposição à radiação solar. Novamente, a nota fornecida a este item, no presente trabalho, não corresponde diretamente à quantidade de exposição solar à qual os entrevistados estão submetidos, e sim como eles julgam a sua própria exposição, se adequada ou não. A percepção da exposição solar, assim, nos pós-graduandos apresentou valores médios de 7,51. Capacitação técnica para orientar adequadamente em relação à exposição solar ou de outras fontes de raios ultravioletas obteve média pelos alunos da pós-graduação de 8,71 e interesse de valor na média de 7,20.

Dados relacionados a ações de prevenção de acidentes e exposição a violência, e controle check up são itens bem menos estudados no que diz respeito à qualidade de vida. Não foram encontrados trabalhos que se referissem a esses itens em entrevistas, em estudos epidemiológicos, e relacioná-los a promoção da saúde, e prevenção de doenças, desta forma, não foi possível relacioná-los e compará-los com os dados do presente estudo. Cuidados em relação à prevenção de acidentes e precaução de situações de violência foram avaliados, e mostraram médias pelos pós- 
graduandos de 7,75 atualmente e 8,96, daqui a um ano. As notas dadas associadas ao check up foram em média 8,65 para a satisfação na realização desses exames. As médias para os alunos pós-graduandos foram para o check up de capacitação, 7,4 e interesse, 7,5.

Orientações que visam prevenir acidentes e evitar situações que possam gerar violência podem ser atribuições médicas. A capacitação técnica para fazê-las foi avaliada pelos alunos da pós-graduação, apresentando média de 6,79 e, interesse no assunto, foi julgado por meio da média de 6,55 com relação a capacitação a nota média foi de 6,3 e 6,1 e interesse de 8,0 e 7,3.

Comparando as médias obtidas neste estudo com as notas apresentadas na aplicação do questionário em alunos do quinto ano da graduação e residentes de clínica médica do primeiro ano, observamos, de maneira geral, diferenças importantes que permitem aventar hipóteses interessantes sobre o comportamento do médico no decorrer de sua formação profissional.

As médias menores dos alunos da pós-graduação se apresentaram, nas questões de comportamento pessoal, nos itens uso de cigarro e drogas, situações de estresse, comportamento sexual, qualidade do sono, vacinação e check $u p$ atuais, sendo que a expectativa para o próximo ano se manteve menor nos itens uso de drogas, estresse e prevenção de acidentes e violência. Em posse de conhecimento sobre os malefícios do cigarro, consequências e efeitos das drogas de abuso, medidas de prevenção de doenças sexualmente transmissíveis e gravidez indesejada, notas mais baixas nesses itens revelam a falta de importância atribuída a esses itens pelos próprios médicos. As questões de atitude profissional, todos os itens abordados (alimentação, bebida alcoólica, cigarro, drogas, atividade física, situações de estresse, comportamento sexual, higiene bucal, sono, exposição ao sol, prevenção de acidentes e violência, check up e vacinação) foram associados a notas mais baixas na avaliação do interesse em informar, e conscientizar os pacientes durante os atendimentos médicos, o que reflete nos pacientes o valor que se dá aos contingentes de promoção à saúde.

As notas maiores entre os pós-graduandos se apresentaram nas questões de comportamento pessoal, relacionadas à alimentação e higiene bucal, tanto no momento

\section{REFERÊNCIAS}

1. Mendes JMR, Wünsch DS. Elementos para uma nova cultura em segurança e saúde no trabalho. Rev Bras Saude Occup. 2007;32(115):153-63. Disponível em: http://www.scielo.br/ $\mathrm{pdf} / \mathrm{rbso} / \mathrm{v} 32 \mathrm{n} 115 / 14$.

2. Serxner SA, Gold DB, Grossmeier JJ, Anderson DR. The relationship between health promotion and medical costs: a dose response. J Occup Environ Med. 2003;5(11):1196-200. DOI: 10.1097/01.jom.0000095002.12772.6a.

3. Ferreira Junior M, Germani ACCG, Pino-Oliveira AA. atual como a expectativa para daqui um ano. Atualmente, as notas maiores isoladas foram apenas às atribuídas à prevenção de acidentes e violência. As questões que versam sobre atitude profissional tiveram notas dos pós-graduandos maiores no item Capacitação, ou seja, sobre todos os temas, os alunos da pós-graduação se julgam mais capacitados em informar e orientar seus pacientes.

Nos demais itens, as notas dos alunos da graduação e da residência médica, e dos alunos da pós-graduação foram iguais (relacionadas, todas, ao comportamento pessoal- momento atual de atividade física, e exposição ao sol, e expectativa para o próximo ano em relação ao uso de cigarro).

Quando comparadas as médias dos alunos da pósgraduação no chamado "Questionário de avaliação de conhecimento", todos os itens apresentaram valores menores em relação às notas de internos e residentes. O conteúdo das informações transmitidas aos pacientes relacionadas à promoção da saúde e prevenção de doenças, portanto, está piorando no decorrer do tempo de formação médica dos alunos. O desinteresse em orientar sobre o tema, constatado no "Questionário de atitudes e comportamento", categoricamente menor nos alunos da pós-graduação, aliado ao auto julgamento de plena capacitação em transmitir essas informações, com médias maiores em relação às dos internos e residentes, podem justificar a pouca importância que se dá ao tema e aparente falta de atualização nos dados específicos a ser abordados de maneira precisa durante atendimento médico.

\section{CONCLUSÃO}

De acordo com os resultados obtidos, e as reflexões cabíveis relacionadas às médias isoladas e a comparação feita com estas, com o estudo anterior, com alunos residentes e internos, observamos certo menosprezo com os temas relacionados a promoção da saúde. O prejuízo na qualidade do atendimento é inegável, uma vez que não há consciência por parte dos entrevistados da própria falta de conhecimento técnico atualizado no conteúdo da orientação sobre promoção da saúde que contrasta com a auto-percepção de capacitação adequada para fazê-lo.

Self perception of medical students and residents regarding personal habits, skills and interest on health promotion and disease prevention. Rev Soc Bras Clin Med. 2011;1(2):1726.

4. Ferreira Junior M, Germani ACCG, Pino-Oliveira AA. Assessing the health promotion and disease prevention knowledge of medical students and residents. Rev Soc Bras Clin Med. 2011;1(2):7-16.

5. Fogaça MC, Carvalho WB, Nogueira PCK, Martins LAN. 
Estresse ocupacional e suas repercussões na qualidade de vida de médicos e enfermeiros intensivistas pediátricos e neonatais. Rev Bras Ter Intens. 2009;21(3):299-305. http:// dx.doi.org/10.1590/S0103-507X2009000300010.

6. Torres AR, Ruiz T, Müller SS, Lima MCP. Qualidade de vida e saúde física e mental de médicos: uma autoavaliação por egressos da Faculdade de Medicina de Botucatu - UNESP. Rev Bras Epidemiol. 2011;14(2):264-75. http://dx.doi.org/10.1590/ S1415-790X2011000200008.

7. Fidalgo TM, Silveira DX. Uso indevido de drogas entre médicos: problema ainda negligenciado. J Bras Psiquiatr. 2008;57(4):267-9. http://dx.doi.org/10.1590/S004720852008000400007.

8. Mirra AP, Rosemberg J. Inquérito sobre prevalência do tabagismo na classe médica brasileira. Rev Assoc Med Bras. 1997;43(3):209-16. http://dx.doi.org/10.1590/S010442301997000300008.

9. Macedo PCM, Cítero VA, Schenkman S, Nogueira-Marins CF, Morais MB, Nogueira-Martins LA. Preditores de qualidade de vida relacionada à saúde durante a residência médica em uma amostra randomizada e estratificada de médicos residentes. Rev Bras Psiquiatr. 2009;31(2)119-24. http://dx.doi.org/10.1590/ S1516-44462009000200007.

10. Silva RS, Silva I, Silva RA, Souza L, Tomast E. Atividade física e qualidade de vida. Ciên Saúde Coletiva. 2010;15(1):115-20. http://dx.doi.org/10.1590/S1413-81232010000100017.

11. Martinez MC, Latorre MRDO, Fischer FM. Capacidade para o trabalho: revisão de literatura. Cien Saude Coletiva. 2010;15:1553-61. http://dx.doi.org/10.1590/S141381232010000700067 .

12. Tirico PP, Stefano SC, Blay SL. Qualidade de vida e transtornos alimentares: uma revisão sistemática. Cad Saude Publica. 2010;26(3):431-49. http://dx.doi.org/10.1590/S0102$311 \times 2010000300002$.
13. Williams CD, Schouten R. Assessment of occupational impairment and disability from depression. JOccup Environ Med. 2008;50(4):441-50. doi: 10.1097/JOM.0b013e318169ccd0.

14. Bianco VC, Lopes ES, Borgato MH, Silva PM, Marta SN. O impacto das condições bucais na qualidade de vida das pessoas com cinquenta ou mais anos de vida. Cien Saúde Coletiva. 2010;15(4):2165-2172. http://dx.doi.org/10.1590/S141381232010000400030 .

15. Müller MR, Guimarães SS. Impacto dos transtornos do sono sobre o funcionamento diário e a qualidade de vida. Estudos Psicol. 2007;24(4):519-28. http://dx.doi.org/10.1590/S0103166X2007000400011.

16. Lourenção LG, Moscardini AC, Soler ZASG. Saúde e qualidade de vida de médicos residentes. Rev Assoc Med Bras. 2010;56(1):81-91. http://dx.doi.org/10.1590/S010442302010000100021.

17. Fontes A, Figlie NB, Laranjeira R. O comportamento de beber entre dependes de álcool: estudo de seguimento. Rev Psiq Clin. 2006;33(6):304-12. http://dx.doi.org/10.1590/S010160832006000600003 .

18. Firth-Cozens J. Doctors, their wellbeing, and their stress: it's time to be protective about stress and prevent it [editorial]. Brit Med J. 2003;326:670 apud Torres AR, et al., 2006.

19. Kurokawa NE, Oliveira LA, Figueiredo WS, Landroni MAS, Waldman CCS, Ayres JRCM. Limites do trabalho multiprofissional: estudo de caso dos centros de referência para DST/Aids. Rev Saude Publica. 2002;36(4 supl):108-16. http:// dx.doi.org/10.1590/S0034-89102002000500015.

20. Cabrera SEM, Merege CES. Inquérito vacinal de alunos de graduação em medicina e enfermagem da Faculdade de Medicina de São José do Rio Preto (SP, Brasil) nos anos de 2006 e 2007 e suas possíveis implicações na atuação discente. Cien Saude Coletiva. 2011;16(2):547-52. http://dx.doi.org/10.1590/ S1413-81232011000200018. 